\title{
On the Implementation of Abstract Interpretation Systems for (Constraint) Logic Programs ${ }^{\star}$
}

\author{
Gerda Janssens and Wim Simoens ${ }^{\star \star}$ \\ K.U. Leuven, Department of Computer Science \\ Celestijnenlaan 200A, B-3001 Heverlee, Belgium \\ \{gerda,wims\}@cs.kuleuven.ac.be
}

\begin{abstract}
We report on an experiment with two tools for global analysis of logic programs. Both systems implement a top-down framework for abstract interpretation. PLAI (Programming in Logic with Abstract Interpretation) developed by Hermenegildo and Muthukumar is written in Prolog. GAIA (Generic Abstract Interpretation Algorithm) developed by Le Charlier and Van Hentenryck is written in C. With each system we have developed two applications. As abstractions we used a domain mode for logic programming and a domain freeness for constraint logic programming over numerical and unification constraints.

Our results allow implementors to make a more knowledgeable choice between both systems and identify a number of practical issues (e.g. normalisation and optimisations) that arise in implementing abstract interpretation systems. The comparison of the results for modes and freeness illustrates the impact of the complexity of abstractions on the performance of the analysis. Moreover, as our experiments use state of the art technology, they give an idea of the feasibility of realising practical abstract interpretation tools based on these and similar abstractions. To our knowledge, this is the first paper that reports on coupling the same abstract domain with abstract interpretation algorithms that use C (GAIA) and Prolog (PLAI).
\end{abstract}

\section{Introduction}

Abstract interpretation [7] is widely used for the global analysis of logic programs and, more recently, also for Constraint Logic Programming [4, 5]. Frameworks have been developed, applications have been discussed and experimental results have been reported by many authors (see [8] for references). This work is motivated by the need for optimisation in LP compilers to close the gap

* This work was funded in part by ESPRIT project 5246 PRINCE and by Diensten voor de Programmatie van het Wetenschapsbeleid (project RFO-AI-02).

** The first author is a post-doctoral researcher of the Belgian National Fund for Scientific Research. Part of the research reported in this paper was performed while the second author was visiting Brown University. 
with procedural languages. Recently two generic algorithms, GAIA (Generic Abstract Interpretation Algorithm) developed by Le Charlier and Van Hentenryck $[13,14]$, and PLAI (Programming in Logic with Abstract Interpretation) developed by Hermenegildo and Muthukumar [18, 19], became widely available. Both algorithms are fine tuned implementations inspired by the top-down framework of Bruynooghe $[2]^{3}$. While these systems differentiate regarding some design decisions, the most noticeable difference is that GAIA is written in C, while PLAI is in Prolog. Researchers interested in using these generic algorithms are confronted with the choice between both systems, between the relative ease of programming the application specific operations in Prolog and the more demanding task of programming them in $C$, with the expectation of obtaining a better performance.

In this paper we report on an experiment where two applications have been developed in both systems. We identify a number of practical issues that arise in designing and implementing abstract interpretation systems (e.g. normalisation and optimisations). Our results show that the real performance gap between both systems is much larger than the factor $5-10$ which is reported by previous Prolog - C comparisons $[17,21]$, namely for small programs performing similar algorithms on similar data structures. An important reason is that $\mathrm{C}$ allows to use very different data structures for representing the elements of the abstract domain, and consequently also very different algorithms for realising the abstract operations upon these elements. This has a major impact on memory consumption and performance. The complexity of the abstraction has a serious impact on the performance of the analysis. Although much slower, it was no problem to do a simple analysis of quite large programs with PLAI. Experimenting with a complex analysis is also feasible, however the memory consumption and execution time of the abstract operations become dominating and may cause problems when analysing large programs. So one can state that PLAI is adequate for developing experimental prototypes but not for integration in a production compiler. For the latter task, GAIA is much better suited and one can expect the extra effort to be rewarded with substantial speedups $(10-100)$. The results obtained for freeness give an idea of the feasibility of such complex domains in production compilers.

Our results dispute the claims of Tan and Lin [20], which report a performance improvement of 100 on a simple domain by compiling the abstract interpretation into the framework of the WAM, instead of the usual interpreter-based approach. Our experiments suggest that the difference is not only due to the effect of compilation, but also to the fact that their interpreter-based analysis is done in Prolog and the compiled analysis in C.

The rest of this paper is organised as follows. In the next section, we review the abstract interpretation systems GAIA and PLAI. Section 3 gives an overview of the abstract domains used in the experiments. Section 4 presents the benchmarks used. Section 5 describes our experiments and compares GAIA and PLAI on the two domains while Section 6 contains the conclusions of this research.

${ }^{3}$ GAIA can also be viewed as an instance of a universal fixpoint algorithm instantiated to a fixpoint semantics for logic programming. 


\section{The Abstract Interpretation Systems}

This section discusses informally some algorithmic and implementation aspects of the abstract interpretation systems that we found relevant for our experiments. We assume some familiarity with top-down frameworks for abstract interpretation of logic programs, such as [2].

\subsection{Overview of the Approach}

As both GAIA and PLAI can be viewed as inspired by the abstract interpretation framework for logic programs of Bruynooghe [2], we briefly recall some basic ideas of this framework. It closely mimics the procedural semantics and gives detailed information about call and success substitutions of the predicates: it constructs an AND-OR graph for a given program and a given query specification. This information can be used for example for further optimisations of the analysed program $[17,21]$.

A generic abstract interpretation system consists mainly of two parts:

- The domain-dependent operations are the abstract operations that need to be implemented in order to instantiate the system to a specific domain.

- The fixpoint algorithm computes abstract substitutions for each program point in the analysed program. These abstract substitutions can be used to construct the AND-OR graph of Bruynooghe's framework.

The key ideas behind top-down abstract interpretation can be summarised as follows. To solve a query to a procedure $p$ with an abstract substitution $\beta$, the algorithm considers each clause defining $p$ and groups the result of the clause analyses using an abstract operation (this is the so-called OR-node of Bruynooghe's framework). The analysis of a clause considers each goal in turn applying the same algorithm to each goal with the abstract substitution obtained for the goal (this is the so-called AND-node of Bruynooghe's framework). Recursion is handled in a special way to guarantee correctness while preserving the safeness of this analysis. When a recursive call is encountered with the same abstract substitution, no new subcomputation is initiated. Rather a lower approximation of the result of this call is returned for the recursive call. The initial lower approximation can simply be the bottom element of the domain or can be computed in a more sophisticated way. The approximation may be updated when a call collects the results of its clauses. If the used approximation has been updated and if the call depends on itself recursively, a new analysis is required. The overall computation is thus an iterative process which terminates when none of the approximations used in the computation have been updated during the last iteration. This framework can be used both for logic programs and constraint logic programs. For CLP the notions of unification and substitutions are generalised to those of conjunction and constraints [9].

The efficient algorithms on which GAIA and PLAI are based aim at optimising the top-down computation of the AND-OR graph. Although the computation is organised around the AND-OR graph, it is not stored as such. Both algorithms gather call and success substitutions for the predicates in the program. They 
both optimise the computational cost of the fixpoint computation, e.g. by avoiding to use bottom as the first approximation of the success substitution of a recursive call, by reducing the computational effort which is performed during the successive iterations in computing the success substitution of a recursive call, and by using the already obtained results when it happens that a predicate is called several times with the same call substitution.

In the rest of this section we discuss differences between them that we found relevant for our experiments.

\section{$2.2 \quad$ Interface}

Normalisation. An important difference between them affects the domain dependent part and is related to the approach towards normalisation. A number of operations are simpler when the arguments of the heads and the calls are variables. For example, the normalisation of the append $/ 3$ program is as follows: append $(X, Y, Z):-X=n i l, Y=Z$.

append $(X, Y, Z):-X=\left[E \mid X^{\prime}\right], Z=\left[E \mid Z^{\prime}\right], \operatorname{append}\left(X^{\prime}, Y, Z^{\prime}\right)$.

The effect of normalisation is to make the unifications explicit, isolating them from the other abstract operations, and to introduce extra variables. How desirable normalisation is depends on the application. Isolating the unifications can make other operations substantially simpler, however, having the extra variables can substantially increase the size of the abstract substitutions in the program points (the size can be exponential in the number of clause variables for some domains). Note also that normalisation can decrease precision. With PLAI, users can decide whether to use normalisation or not, GAIA requires strong normalisation, unifications must be of the form $X=Y$ or $X=f\left(Y_{1}, \ldots, Y_{n}\right)$ with $X, Y, Y_{1}, \ldots Y_{n}$ distinct variables. The possible loss of precision due to the weakness of information regarding the dependencies between variables which is stored in the abstractions can be compensated by the reexecution strategy $[2,15]$. However, the introduction of extra variables remains and can be a problem in some applications. Therefore, GAIA has been slightly modified to allow weaker forms of normalisation. The modified GAIA system assumes that the input programs fulfill some minimal normalisation requirements, namely atoms are of the form $p\left(X_{1}, \ldots, X_{i}\right)$ and the arguments are distinct variables. The unifications still have to be explicit, but the application decides about their actual form. In our experiments GAIA and PLAI analyse identical programs in weak normal form.

Representation of Variables. Another difference is the internal representation of the program, in particular the representation of the variables in the source program. In GAIA the program variables are represented by numbers i.e. $0,1, \ldots$, while PLAI represents program variables by free Prolog variables. The difference is important for the choice of the data structure for the abstract substitutions. Note that abstract substitutions often contain sets of variables (see also the high level description of the two abstract domains in Section 3). In GAIA, this is a set of numbers (between 0 and $n$ ) for which a bit representation is a compact and reasonably efficient representation in C. In PLAI this is a set of free variables that 
can be represented by an ordered list in Prolog, but this representation consumes more memory and requires more time to process. The variable representation in PLAI allows the use of Prolog specific implementation techniques, e.g. using unification to rename abstract substitutions, but bit operations cannot be used with this free variable representation.

\subsection{Fixpoint Algorithm}

Avoiding Redundant Computation. In the treatment of a recursive predicate, the Original version of GAIA recomputes all clauses defining the predicate. PLAI does better: it recomputes only the clauses from which the predicate is reachable. Both systems redo the unifications, however they avoid to recompute calls to predicates for which the call pattern has not changed. They simply pick up the success substitution stored in the previous iteration. GAIA has a variant, the Prefix version which is theoretically more efficient, as it starts recomputation from the point where the recursive call receives a new success substitution (to allow this the abstract substitution in the program point prior to the recursive call is stored, the abstract substitution of other program points is not stored). For example, PLAI and the Original version of GAIA will reexecute the unifications in the recursive clause of append, while the Prefix version of GAIA will not. In practice, it is not always the case that the Prefix version is better as the extra cost of storing and fetching the full substitution in the point prior to the recursive call can be more substantial than the cost of recomputing the calls prior to the recursive call.

Granularity of the Analysis and Annotation. The analysis can derive different call patterns for a procedure, e.g. the same procedure can be called from different points in the program or recursive calls may have other call patterns than the original one. Both systems compute dynamically as many versions, i.e. call patterns, for a procedure as encountered during fixpoint computation. The systems have a different approach towards presenting the computed AND-OR graph to the user. GAIA outputs all versions explicitly, while PLAI gives one version - the most general one - for each predicate. A version is annotated with abstract sub-. stitutions in the program points. PLAI keeps the abstract substitution in every program point (so that it can be used directly to annotate the program), while GAIA only keeps the call and success patterns for the predicate calls. So that at the moment of annotation, the AND-OR graph has to be partly recomputed in GAIA. This post-processing step of GAIA is straightforward and linear in the size of the programs and has the advantage of reducing the storage of information which may not be relevant during the fixpoint computation. Apparently, the design decision of PLAI slows it down as there is the overhead of storing this information (i.e. copying these substitutions in Prolog's record database).

\subsection{Implementation Language}

In addition to the previous aspects, when one tries to compare GAIA and PLAI, one has to take into account the well known differences between the procedural 
programming paradigm and the logic programming paradigm. PLAI uses Prolog and this allows rapid prototyping. This is especially advantageous while one is still experimenting with the expressive power of the abstract domain. In the case of GAIA, one has to implement the abstract domain in C and this seems only worthwhile once a definite decision about the abstraction has been made. On the other hand, there remains the performance gap between Prolog and $C$ for which a factor of at most 10 is put forward in [17, 21] for some small programs using similar data structures, but which can be much higher when the $\mathrm{C}$ version encodes the information in a different way and processes it with different algorithms (cf. different internal representation of variables).

\section{The Abstract Domains}

We have coupled GAIA and PLAI with two abstract domains. The abstract operations are defined for (weakly) normalised programs, although this is not required by PLAI. Each domain has been implemented in $\mathrm{C}$ and in Prolog. For each language we took an appropriate data structure that allowed to use the full power of the language. It is the intention of the authors that the implementation of the domains allows a fair comparison between the two systems.

These two domains are very different from a complexity viewpoint and enable us to study the impact of normalisation and sizes of the abstract substitutions on the systems. In addition, they give interesting data on the feasibility of other domains such as complex sharing domains.

The domain mode aims at deriving instantiation states of program variables in logic programs. The domain mode is also used in [13] and is a reformulation of the domain in [2]. An abstract substitution has three components: (1) the mode component associates the mode var, ground or any with each program variable, (2) the sval component defines an equivalence relation between program variables that definitely have the same value, and (3) the sharing component defines an equivalence relation between program variables that possibly share a variable. The abstraction of $\left\{X=f\left(Y_{1}, Y_{2}\right), Y_{1}=T\right\}$ is the triplet: ( $\left\{\right.$ mode $(X$, any $), \operatorname{mode}\left(Y_{1}\right.$, var $\left.), \operatorname{mode}\left(Y_{2}, \operatorname{var}\right), \operatorname{mode}(T, v a r)\right\},\left\{\{X\},\left\{Y_{1}, T\right\}\right.$, $\left.\left.\left\{Y_{2}\right\}\right\},\left\{\left\{X, Y_{1}, Y_{2}, T\right\}\right\}\right)$. The set $\left\{Y_{1}, T\right\}$ in the sval component expresses that $Y_{1}$ and $T$ have the same value. The set $\left\{X, Y_{1}, Y_{2}, T\right\}$ in the sharing component expresses that the four variables might have a common variable.

The freeness domain $[11,12]$ derives information about definite freeness of program variables in constraint systems. It deals with mixed constraint systems in CLP programs, namely systems containing numerical constraints, e.g. $Y_{1}+T=3$, and unification constraints, e.g. $X=f\left(Y_{1}, Y_{2}\right)$. The abstraction extends the notion of possible sharing in the context of LP towards possible dependencies established by constraint systems in CLP. In order to decide about definite freeness of a variable the abstraction must capture the dependencies that are created by the constraint system. Then we can safely compute which variables might get instantiated - and are possibly not free - when a particular variable gets a value. The abstraction has again a mode compo- 
nent and a set of sets of variables describing a.o. possible dependencies. The constraint system $\left\{X=f\left(Y_{1}, Y_{2}\right), Y_{1}+T=3\right\}$ is abstracted by the tuple: ( $\{$ mode $(X$, any $)$, mode $(Y 1$, var $)$, mode $(Y 2, v a r), \operatorname{mode}(T, v a r)\},\left\{\{X\},\left\{X, Y_{1}\right\}\right.$, $\left.\left.\left\{X, Y_{2}\right\},\left\{Y_{1}, T\right\},\{X, T\},\left\{X, Y_{1}, Y_{2}\right\},\left\{X, Y_{1}, T\right\},\left\{X, Y_{2}, T\right\},\left\{X, Y_{1}, Y_{2}, T\right\}\right\}\right)$.

A variable, e.g. $T$, has mode var as long as $\{T\}$ does not appear in the second component. The value of $T$ depends on $Y_{1}$ and via $Y_{1}$ the value of $T$ also depends on $X$. The sets $\left\{Y_{1}, T\right\}$ and $\{X, T\}$ express these (possible) dependencies. Note that the set of sets is closed under union. The technical reasons for this are beyond the scope of this paper (see [12]).

For this paper it is important to notice the difference in space requirements of the abstractions. mode requires quadratic space in the number of variables in the worst-case because of the sharing component on pairs of variables. freeness may require exponential space since it maintains sets of sets of variables to express sophisticated dependencies between variables. Hence its size is very sensitive to the introduction of extra variables by normalisation. Note that [10] describes two ways to reduce the size of the freeness domain (by combining it with definiteness information [9] and by using a more compact representation for the information).

\section{Benchmarks}

The programs we use for the simple mode domain are the same as in [13]. They are "pure" logic programs and used for various purposes from compiler writing to equation-solvers, combinatorial problems and theorem-proving. Hence they should be representative of a large class of programs.

For the freeness domain, we have chosen CLP specific examples. Due to the intrinsic size of the abstract substitutions, we had to restrict the size of the benchmark programs. Lengthlist and Sumlist compute resp. the length and the sum of the elements of a given list. Meal is a program to compute balanced meals. Vecadd is a program to compute the sum of two vectors (as lists). Matvec computes the multiplication of a matrix with a vector and Matmul is a program for the multiplication of two matrices. Another famous program is the mortgage program. We used it with two different call-patterns (mg1 and mg2 for short. The relevant difference between them is that mg2 gives rise to two call patterns, while mg1 only to one). Runge-kutta and trapezoid encode integration methods to solve first order ordinary differential equations. The programs are taken from the Prolog III example programs or programs shared by the PRINCE partners.

\section{Experiments}

This section reports upon our experience gained in using GAIA and PLAI when coupling them with two different abstract domains. Based on the experiments we draw some conclusions which can help developers to make a more knowledgeable choice between the two systems. First, we investigate the behaviour of GAIA when coupled with different domains. Then, we compare the abstract 
interpretation applications developed with GAIA and PLAI. Finally, an overview is given of points which our experiments showed to be important for the developer of abstract interpretation applications. The experiments are done on a SUN SPARC2 and the execution times (CPU times) are given in seconds. PLAI is compiled using SICStus 2.1 \#3.

\subsection{On Coupling GAIA with Different Domains}

The GAIA-system implements two different algorithms of [13]: the Original version and the Prefix version. They differ in the kind of optimisations used during the successive iterations of the fixpoint computations as explained in Section 2.3.

Table 1. Execution time of GaIA coupled with the mode domain

\begin{tabular}{|c|c|c|c|c|}
\hline Program & Or & \multicolumn{2}{|c|}{$\overline{\mathrm{Pr}} \mathrm{Pr} / \mathrm{Or} \%$} & \\
\hline append & 0.01 & 0.00 & 0.00 & the append program \\
\hline cs & 5.86 & 3.02 & 51.53 & cutting stock problem \\
\hline disj & 2.08 & 0.81 & 38.94 & solve disjunctive scheduling problem \\
\hline gabriel & 0.77 & 0.36 & 46.75 & browse program from gabriel benchmark \\
\hline Kalah & 1.55 & 0.79 & 50.96 & the game of kalah \\
\hline peep & 2.60 & 1.18 & 45.38 & peephole optimisation in SB-prolog compiler \\
\hline $\mathrm{p} 8$ & 0.33 & 0.14 & 42.42 & specific mathematical problem by W. Older \\
\hline plan & 0.20 & 0.09 & 45.00 & planning program from Sterling \& Shapiro \\
\hline press & 3.66 & 1.73 & 47.26 & equation solver program \\
\hline qsort & 0.22 & 0.09 & 40.90 & the quicksort program \\
\hline queens & 0.10 & 0.05 & 50.00 & the n-queens program \\
\hline read & 3.75 & 1.54 & 41.06 & tokeniser/reader by O'Keefe and Warren \\
\hline Average & & & 45.47 & \\
\hline
\end{tabular}

Our Starting Point. The behaviour of the Prefix version with respect to the Original version is very different for our abstract domains. The execution times for mode are given in Table 1: the first column (Or) gives the time of the Original version and the second column ( $\mathrm{Pr}$ ) the time of the Prefix version (we used the same mode domain and actually the same C-code as in [13]). For freeness the same information is given under the heading DS1Strong in Table 2. In case of the mode domain, the Prefix version needs on the average only $45 \%$ of the time of the Original version, while for the freeness domain this is $381 \%$. This is partly due to the effect of the intrinsic size of the freeness domain on the fixpoint optimisations of the Prefix version, since freeness is no longer polynomial in the worst-case as mode is, but rather exponential. However, two other causes, namely the choice of the data structure for the freeness domain and the degree of normalisation, have been identified.

The Impact of Data Structures. We used the following data structures for the set of sets of variables in the freeness domain:

1. First, a red-black tree [1] (balanced binary tree), where each node was extended with a pointer to the next in-order element (DS1) has been chosen 
Table 2. Execution times of GAIA coupled with the freeness domain: influence of the data structure on the behaviour of GAIA

\begin{tabular}{|c|c|c|c|c|c|c|}
\hline \multirow{2}{*}{$\frac{\text { Program }}{\text { sumlist }}$} & \multicolumn{3}{|c|}{ 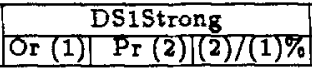 } & \multicolumn{3}{|c|}{\begin{tabular}{|c|} 
DS2Strong \\
Or $_{\mathrm{r}}(3) \operatorname{Pr}(4)[(4) /(3) \%$ \\
\end{tabular}} \\
\hline & 0.11 & 0.14 & 127.27 & 0.14 & \begin{tabular}{|l|l|}
0.13 \\
\end{tabular} & 92.86 \\
\hline lengthlist & 0.05 & & & 0.05 & & \\
\hline meal & 0.97 & & 161.86 & 0.38 & 0.45 & 118.42 \\
\hline & & & & 6.43 & & 89.42 \\
\hline matre & 4.81 & & 04.78 & 4.93 & 4.4 & 39.45 \\
\hline $\mathrm{mg} 1$ & 38.70 & 364.54 & 941.96 & 38.77 & 36.45 & 94.02 \\
\hline $\mathrm{mg}^{2}$ & 187.37 & 1654.11 & 882.80 & 188.11 & 186.63 & 99.21 \\
\hline Average & & & 381.60 & & & 97.63 \\
\hline
\end{tabular}

because it allows fast and easy binary search for the set of sets and the redblack colouring convention keeps the tree balanced. The pointer was used to have an ordered list representation to speed-up the implementation of some of the abstract operations. Profiling both versions showed that the time needed in the Prefix version to store and fetch the information for the fixpoint optimisation is bigger than the time needed by the Original version to do the whole computation. For example in the case of mg1, the time used for the Prefix optimisations is a factor 5.2 of the time to redo the computations shortcut by the optimisations. The Prefix optimisations require copying of abstract substitutions. In the case of freeness this is expensive because of the intrinsic exponential size of the abstract substitutions. Moreover, copying is complicated by the pointer to the next in-order element.

2. To speed-up the copy operation, we removed the pointer to the next in-order element and used just a red-black tree to represent the set of sets of variables (DS2). Now, the time needed to copy an abstract substitution dropped with more than $90 \%$ and the Prefix version is on the average $2.5 \%$ faster than the Original version (see part of Table 2 under heading DS2strong).

In the mode domain, the time needed to store (or fetch) an abstract substitution is almost negligible: the size of a substitution is polynomial and the used data structure allows a very fast copy, so here the performance of the Prefix version is much better than the Original version.

The Impact of Degree of Normalisation. By degree of normalisation we mean the kind of restrictions put on the allowed forms for unification and numeric constraints. The stronger the normalisation, the more extra variables are used: e.g. $X=f(A, B), A=h(Y), B=1$ is the strongly normalised form of $X=$ $f(h(Y), 1)$. The effect of the degree of normalisation can best be shown through some examples using the freeness domain: here the size of the abstract substitutions is exponential with respect to the number of variables in the clause: e.g. (1) for sumlist, introducing one extra variable causes the time to be doubled, and (2) if in both the first and second clause of mortgage five extra variables are introduced, the time needed in GAIA (and PLAI) was multiplied with at least a 
Table 3. Execution times of GAIA coupled with the freeness domain: effect of normalisation on DS1

\begin{tabular}{|c|c|c|c|c|c|c|}
\hline Program & \begin{tabular}{|c|} 
DS1S \\
Or (1) \\
\end{tabular} & \begin{tabular}{|l|} 
trong \\
Pr (2)
\end{tabular} & \begin{tabular}{|c|} 
DS1V \\
Or $(3)$ \\
\end{tabular} & $\begin{array}{l}\text { Veak } \\
\text { Pr (4) }\end{array}$ & $\|(3) /(1) \%$ & $(4) /(2) \%$ \\
\hline sumlist & 0.11 & 0.14 & 0.02 & 0.02 & 18.18 & 14.29 \\
\hline lengthlist & 0.05 & 0.05 & 0.02 & 0.02 & 40.00 & 40.00 \\
\hline meal & 0.97 & 1.57 & 0.01 & 0.01 & 1.03 & 0.64 \\
\hline vecadd & 6.23 & 15.73 & 1.16 & 1.37 & 18.62 & 8.71 \\
\hline matrec & 4.81 & 9.85 & 1.43 & 1.39 & 29.73 & 14.11 \\
\hline matmul & 509.37 & OOM & 19.44 & 35.35 & 3.82 & 1 \\
\hline $\mathrm{mg} 1$ & 38.70 & 364.54 & 1.19 & 0.92 & 3.07 & 0.25 \\
\hline mg2 & 187.37 & 1654.11 & 3.65 & 4.24 & 1.95 & 0.26 \\
\hline runge-kutta & $\overline{\mathrm{OOM}}$ & OOM & 363.75 & 451.43 & 7 & 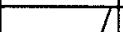 \\
\hline trapezoid & OOM & OOM & 618.70 & $\mathrm{OOM}$ & $\pi$ & 7 \\
\hline Áverage & & & & & 14.55 & 11.18 \\
\hline
\end{tabular}

Table 4. Execution times of GAIA coupled with the freeness domain: effect of normalisation on DS2

\begin{tabular}{|c|c|c|c|c|c|c|}
\hline Program & \begin{tabular}{|c|} 
DS2S \\
Or (1) \\
\end{tabular} & $\begin{array}{l}\text { rong } \\
\text { PI (2) }\end{array}$ & \begin{tabular}{|c|} 
DS22 \\
Or (3)
\end{tabular} & $\begin{array}{l}\text { Weak } \\
\text { Pr (4) }\end{array}$ & $\|^{(3) /(1) \%}$ & $(4) /(2) \%$ \\
\hline sumlist & 0.14 & 0.13 & 0.01 & 0.01 & 7.14 & 7.69 \\
\hline lengthlist & 0.05 & 0.05 & 0.01 & 0.01 & 20.00 & 20.00 \\
\hline meal & 0.38 & 0.45 & 0.02 & 0.02 & 5.26 & 4.44 \\
\hline recadd & 6.43 & 5.75 & 1.08 & 0.96 & 16.80 & 16.70 \\
\hline matrec & 4.93 & 4.41 & 1.46 & 0.99 & 29.61 & 22.45 \\
\hline matmul & 527.83 & 486.23 & 19.13 & 16.28 & 3.62 & 3.35 \\
\hline mg1 & 38.77 & 36.45 & 1.10 & 0.86 & 2.84 & 2.36 \\
\hline $\mathrm{mg}^{2}$ & 188.11 & 186.63 & 3.61 & 3.07 & 1.92 & 1.64 \\
\hline runge-kutta & OOM & $\overline{O O M}$ & 357.69 & 367.72 & & 1 \\
\hline trapezoid & OOM & $\mathrm{OOM}$ & 610.92 & 536.58 & 7 & 7 \\
\hline Average & & & & & 10.90 & \begin{tabular}{|l|}
9.83 \\
\end{tabular} \\
\hline
\end{tabular}

factor 60 .

GAIA strongly normalised the programs internally in the system. This caused not only a very bad time performance on the freeness domain, but also memory problems: due to the exponential size of the abstract substitutions, the introduction of only a few extra variables causes a lot of extra memory needed (e.g. we were not able to compute the abstract interpretation of matmul, runge-kutta or trapezoid). Therefore, we modified the GAIA system: the normalisation was removed from the framework and the user can tune the degree of normalisation with respect to his abstract domain (e.g. for our applications the unifications must be of the form $X=t$ with $t$ any term). For the freeness domain, the execution times for this weak normalisation are given in Tables 3 and 4 under the headings DS1Weak and DS2Weak, where OOM stands for Out Of Memory. The performance improves with respect to the strong normalisation for both data structures and both versions with at least $85 \%$. For the mode domain, one can choose to have a strongly normalised input program, as the introduction of a few new variables does not cause time or memory problems. 
Note that the best implementation of freeness with GAIA uses the red-black tree without extra pointer under weak normalisation i.e. DS2Weak in Table 4.

\subsection{Comparison of GAIA and PLAI Based on the Developed Abstract Interpretation Applications}

Table 5. Execution times of GAIA and PLAI coupled with the mode domain

\begin{tabular}{|c|c|c|c|c|c|c|}
\hline & GAIA & & $\overline{\text { PLAI }}$ & & Total/Pr & Anal/Px \\
\hline Program & $\mathrm{Pr}_{\mathrm{r}}$ & Anal & Garb_st & Total & & \\
\hline append & 0.00 & 0.19 & 0.00 & 0.19 & & \\
\hline cs & 3.02 & 104.99 & 25.34 & 130.33 & 43.15 & 34.76 \\
\hline disj & 0.81 & 27.04 & 8.39 & 35.43 & 43.74 & 33.38 \\
\hline gabriel & 0.36 & 14.62 & 3.96 & 18.58 & 51.61 & 40.61 \\
\hline peep & 1.18 & 65.69 & 16.54 & 82.23 & 69.69 & 55.67 \\
\hline $\mathrm{pg}$ & 0.14 & 5.20 & 1.60 & 6.80 & 48.57 & 37.14 \\
\hline plan & 0.09 & 3.83 & 1.27 & 5.10 & 56.67 & 42.56 \\
\hline press & 1.73 & 69.88 & 14.78 & 84.66 & 48.94 & 40.39 \\
\hline qsort & 0.09 & 3.47 & 1.00 & 4.47 & 49.67 & 38.56 \\
\hline queens & 0.05 & 1.57 & 0.90 & 2.47 & 49.40 & 31.40 \\
\hline read & 1.54 & 174.97 & 37.99 & 212.96 & 138.28 & 113.62 \\
\hline Average & & & & & 59.97 & 46.81 \\
\hline
\end{tabular}

Table 6. Execution times of GAI and PLAI coupled with the freeness domain

\begin{tabular}{|c|c|c|c|c|c|c|}
\hline & GAIA & & PLAI & & Total/PI & $\mid$ Anal/Pr \\
\hline Program & $\mathrm{Pr}$ & Anal & Garb_St & Total & & \\
\hline sumlist & 0.01 & 0.17 & 0.00 & 0.17 & .17 .00 & 17.00 \\
\hline lengthlist & 0.01 & 0.15 & 0.00 & 0.15 & 15.00 & 5.00 \\
\hline meal & 0.02 & 0.21 & 0.00 & 0.21 & 0.50 & 0.50 \\
\hline vecadd & 0.96 & 10.23 & 5.74 & 15.97 & 6.64 & 0.66 \\
\hline matvec & 0.99 & 11.31 & 6.69 & 18.00 & 18.18 & 11.42 \\
\hline matmul & 16.28 & 169.52 & 128.53 & 298.05 & 18.31 & 10.41 \\
\hline $\mathrm{mg} 1$ & 0.86 & 8.71 & 5.89 & 14.60 & 16.98 & 10.13 \\
\hline $\mathrm{mg}^{2}$ & 3.07 & 29.92 & 17.42 & 47.34 & 15.42 & 9.75 \\
\hline Funge-ku & \begin{tabular}{|l|}
367.72 \\
\end{tabular} & 1 & & OOM & 1 & \\
\hline trapezoid & 536.58 & 7 & 7 & OOM & ] & \\
\hline Average & & & & & 16.00 & 11.86 \\
\hline
\end{tabular}

GAIA and PLAI are state of the art implementations. Fixpoint optimisations are incorporated in the Prefix version of GAIA and in PLAI. The data structures used to represent the elements of the abstract domain and the algorithms used to process them are very different due to the different nature of the programming languages Prolog and $\mathrm{C}$ and due to the internal representation of the program variables by the systems. What we compare is the performance of both analyses for the same source programs in the same normal form. For PLAI, we give the time needed for the analysis (Anal), for garbage collection and stack shifts (Garb_St) and the total time (Total).

For the mode domain, all benchmarks - including quite large programs - can be run by both systems. However, there is a performance difference of about a 
factor 60 on the average between GAIA and PLAI (cfr. Table 5). For the more complex freeness domain, only small programs can be run with PLAI due to memory problems. The average performance improvement for them is about a factor 16 (cfr. Table 6 ).

The difference between the systems is partly due to the different approaches towards avoidance of redundant computation, annotating programs and granularity of the analysis (as described in Section 2.3) and partly due to the performance gap between Prolog and C. However, we noticed that a substantial amount of the execution time goes into the abstract operations (e.g. about $98 \%$ for the freeness domain coupled to GAIA). So, the difference between GAIA and PLAI is mainly due to the abstract operations and it turns out that for this kind of applications the performance gap between Prolog and $\mathrm{C}$ is bigger than a factor $5-10$ which was put forward for some small programs using similar data structures in $[17,21]$. In our applications, the implementations of the abstract domains use quite different data structures in $C$ than in Prolog. For the freeness domain - and to a lesser degree also for the mode domain - the abstract operations mainly manipulate the sets of sets of variables. They have a much more efficient implementation in $\mathrm{C}$ than in Prolog. A set of variables is represented in GAIA by a bit representation (1 memory cell represents a set of at most 32 variables), while in PLAI an ordered list is used (a simulation of the bit representation cannot be used because the variables in PLAI are represented internally by free variables). For example for a set of 5 elements, 10 times more memory cells are used in Prolog than in C. The abstract operations can be implemented more efficiently for the bit representation than for the ordered lists. The data structures in C can be updated destructively, while in Prolog parts of them have to be copied. Moreover, a program that uses more memory is likely to use more time due to non-locality.

Recently, Tan and Lin [20] have investigated the alternative of compiling abstract interpretation into the framework of the WAM. A prototype analyser based on this idea (written in C) is compared with the analyser in Aquarius [21] (written in Prolog) and gives a performance improvement of a factor of over 150 in the average. Unfortunately, both analysers were coupled to different abstract domains. If the same domain is used, their conservative estimate is a speedup of over 100 for relatively small programs. For mode we obtain a factor 60 as an average for a representative set of programs. However, this is obtained by a meta-interpreting approach for which [20] claims that the compilation into WAM is a promising alternative. Our results seem to contradict their conclusion in the sense that the performance gap between Prolog and $\mathrm{C}$ could be at least as significant as the difference between an interpreter-based and a compiler-based approach for abstract interpretation.

\subsection{Important Issues for Abstract Interpretation Applications}

When choosing a tool to develop an abstract interpretation system, the user should be aware of the following important points: 
1. The approach towards normalisation has an effect on the abstract domain. If the abstract interpretation tool imposes normalisation, one should take this into account during the design of the abstract domain, because its expressive power must be strong enough to obtain the desired precision. If no normalisation is required, the user can choose the degree of normalisation for the application at hand.

The abstract interpretation tool should leave enough freedom to the user w.r.t. normalisation: e.g. PLAI does not require any normalisation and in the version of GAIA we adapted the degree of normalisation is left to the user.

2. The internal representation of the variables affects the choice of the data structure for the abstract substitutions, in particular the data structure to represent a set of variables.

3. The inherent size of the abstract substitutions might interact with the systems approach towards fixpoint optimisations: the behaviour of the Prefix version with respect to the Original version of GAIA is quite different in the case of the freeness domain than in case of the mode domain.

Moreover, the approach towards fixpoint optimisations affects the choice of the data structure for the abstract substitutions. Both abstract interpretation tools store extra information containing abstract substitutions. This involves storing/copying of the abstract substitutions into global data structures. Note that in Prolog this is typically done by using the record data base or using dynamic predicates that are asserted and retracted. Now, putting more (redundant) information in the data structure for the abstract substitutions (i.e. using a smart representation that trades space for time) speeds up the abstract operations, but might slow down the fixpoint optimisations due to their size. This phenomenon was observed during our experiments where the occurrence of a pointer in DS1 complicated the copying operation in GAIA and made the Prefix version slower than the Original one.

The user should be careful when chosing a data structure for the abstract substitutions because the size and the internal organisation (cfr. internal pointers) may interact with the storing/copying of the information done by the fixpoint optimisations.

4. The user should have the option to tune the output of the system towards his needs, e.g. the desired number of versions and the way the results are presented to the user. The flexibility of both systems can be improved.

5. Each programming paradigm has its specific characteristics which are advantageous at different stages of the development of an abstract interpretation system, namely the prototyping phase and the production phase.

6. Neither of the systems offers extra facilities to debug the application under development. One has to rely on the debugging facilities for the underlying language. 


\section{Conclusions}

The paper reports on our experiments of coupling two different abstract domains - modes and freeness - with GAIA and PLAI. An important motivation is the need of turning abstract interpretation into a practical compilation tool. GAIA (written in C) and PLAI (written in Prolog) are both fine tuned implementations of efficient abstract interpretation algorithms. Modes [13] is an instance of a well known application in the context of LP, whereas freeness [12] has recently been proposed in the context of CLP. The two domains are of a different complexity: the intrinsic size complexity of freeness is in the worst case exponential; that of modes polynomial. Freeness can be seen as a representative example of abstractions analysing complex dependencies between program variables.

The paper contains an overview of design decisions in GAIA and PLAI which our experiments showed to be relevant for developers of applications. They are based on different design decisions concerning the interface between the fixpoint algorithm and the abstract domain (normalisation and representation of variables) and concerning the fixpoint algorithm (avoiding redundant computation and annotating programs). Our experiments showed that the performance of the abstract interpretation systems is affected by the degree of normalisation and by the choice of the data structures. An inappropriate choice of the latter can destroy the effect of optimisations that aim at reducing the number of abstract operations, as shown by the experiments on coupling freeness with GAIA. Our experiments illustrate the impact of the complexity of the abstractions on the feasibility of integrating them - and also comparable abstractions - in practical compilation tools. As the performance for freeness is unacceptable for larger programs, the reduction of its size as proposed in [10] is essential to make it practical. Another important observation is that for our experiments most of the execution time is spent on the abstract operations. Therefore, the implementation of the abstract domain turns out to be crucial. In this context, the comparison between systems developed with GAIA and with PLAI boils down to a comparison between $\mathrm{C}$ and Prolog. PLAI has the advantage of supporting rapid prototyping which is interesting while experimenting with the abstract domain. Quite complex domains such as freeness can be run only on example programs. GAIA allows to make implementations of the abstract domains that obtain better performance and consume less memory. In production systems these factors are important to use the system for all kinds of programs. Tan and Lin [20] conclude that a compiler-based approach to abstract interpretation could be a factor 100 better than an interpreter-based one. This difference seems to be due to writing the compiler-based one in C and the interpreter-based one in Prolog, as our experiments reveal the impact of the performance difference between $\mathrm{C}$ and Prolog. Their conclusions hold for an abstract domain which extends the mode domain with type information. Recently, the paper [3] describes a bottom up Prolog implementation for groundness analysis by means of the domain Prop of propositional formulae, which is quite different from the above domains. For this particular domain, the efficiency of [3] is comparable with the $\mathrm{C}$ implementations described in $[6,16]$. 
In order to implement an application with GAIA and PLAI, one has to know how the programs are represented internally in the systems. The main development effort has to be put in the abstract operations. For example, for the freeness domain the difference in development time (including experiments with different data structures) was about a factor 3 .

\section{Acknowledgement}

We are indebted to Maria Garcia de la Banda and Francisco Bueno for making PLA I available and for providing us with the necessary information about the implementation details of PLAI, to Vincent Englebert for coupling the mode domain with PLAI and to Pascal Van Hentenryck and Maurice Bruynooghe for the many valuable comments on the draft versions of this paper.

\section{References}

1. R. Bayer. Symmetric binary B-trees : Data structure and maintenance. Acta Informatica, 1:290-306, 1972.

2. M. Bruynooghe. A practical framework for the abstract interpretation of logic programs. Journal of Logic Programming, 10(2):91-124, Feb. 1991.

3. M. Codish and B. Demoen. Analysing Logic Programs Using "Prop"-ositional Logic Programs and a Magic Wand. In D. Miller, editor, Proceedings of the 1993 International Logic Programming Symposium, pages 114-129, Vancouver, October 1993. MIT Press.

4. J. Cohen. Constraint logic programming languages. Communications of the $A C M$, $30(7): 52-68,1990$.

5. A. Colmerauer. An introduction to PROLOGIII. Communications of the ACM, $30(7): 69-96,1990$.

6. M.-M. Corsini, K. Musumbu, A. Rauzy, and B. Le Charlier. Efficient Bottom-up A bstract Interpretation of Prolog by means of Constraint Solving over Symbolic Finite Domains. In M. Bruynooghe and J. Penjam, editors, Programming Language Implementation and Logic Programming, Lecture Notes in Computer Science 714, pages 75-91, Tallinn, Estonia, 1993. Springer Verlag.

7. P. Cousot and R. Cousot. Abstract interpretation: A unified lattice model for static analysis of programs by construction or approximation of fixpoints. In Proceedings of the Fourth ACM Symposium on Principles of Programming Languages, pages 238-252, Los Angeles, 1977.

8. P. Cousot and R. Cousot. Abstract interpretation and application to logic programs. The Journal of Logic Programming, 13(2 and 3):103-179, 1992.

9. M. G. de la Banda and M. Hermenegildo. A Practical Approach to the Global Analysis of CLP Programs. In D. Miller, editor, Proceedings of the 1993 International Logic Programming Symposium, pages 437-455. MIT Press, October 1993.

10. V. Dumortier and G. Janssens. Towards a Practical Full Mode Inference System for CLP(H,N). Technical Report CW185, Department of Computer Science, Katholieke Universiteit Leuven, Dec. 1993.

11. V. Dumortier, G. Janssens, and M. Bruynooghe. Detection of Free Variables in the Presence of Numeric Constraints. In Proceedings of JICSLP'92 Post-conference workshop on CLP, pages 105-118, 1992. 
12. V. Dumortier, G. Janssens, M. Bruynooghe, and M. Codish. Freeness Analysis in the Presence of Numerical Constraints. In D. S. Warren, editor, Proceedings of the Tenth International Conference on Logic Programming, pages 100-115. MIT Press, June 1993.

13. V. Englebert, B. Le Charlier, D. Roland, and P. Van Hentenryck. Generic Abstract Interpretation Algorithms for Prolog : Two Optimization Techniques and Their Experimental Evaluation. In M. Bruynooghe and M. Wirsing, editors, Proceedings of the 4th International Symposium on Programming Language Implementation and Logic Programming (PLILP 92), pages 311-325, Leuven, 1992. LNCS, Springer Verlag. Also in Software Practice and Experience, Volume 23 No. 4 pp. 419-460, 1993.

14. B. Le Charlier, K. Musumbu, and P. Van Hentenryck. A generic abstract interpretation algorithm and its complexity analysis (extended abstract). In K. Furukawa, editor, Proceedings of the Eighth International Conference on Logic Programming, pages 64-78, Paris, 1991. MIT Press, Cambridge.

15. B. Le Charlier and P. Van Hentenryck. Reexecution in abstract interpretation of prolog (extended abstract). In K. Apt, editor, Proceedings of the Joint International Conference and Symposium on Logic Programming, pages 750-764, Washington, 1992. MIT Press, Cambridge.

16. B. Le Charlier and P. Van Hentenryck. Groundness Analysis for Prolog: Implementation and Evaluation of the Domain Prop. In D. Schmidt, editor, ACM SIGPLAN Symposium on Partial Evaluation and Semantics-Based Program Manipulation, PEPMA'93, pages 99-110, Copenhagen,Denmark, 1993. ACM Press.

17. A. Mariën, G. Janssens, A. Mulkers, and M. Bruynooghe. The impact of abstract interpretation: An experiment in code generation. In G. Levi and M. Martelli, editors, Proceedings of the Sixth International Conference on Logic Programming, pages 33-47, Lisbon, 1989. MIT Press, Cambridge.

18. K. Muthukumar and M. Hermenegildo. Deriving A Fixpoint Computation Algorithm for Top-Down Abstract Interpretation of Logic Programs. Technical Report ACT-DC-153-90, Microelectronics and Computer Technology Corporation (MCC), Austin, TX 78759, Apr. 1990.

19. K. Muthukumar and M. Hermenegildo. Compile-time Derivation of Variable Dependency Using Abstract Interpretation. Journal of Logic Programming, 13(2\&3):315-347, July 1992.

20. J. Tan and I. Lin. Compiling Dataflow Analysis of Logic Programs. In $A C M$ SIGPLAN'92 PLDI, pages 106-115, 1992.

21. P. Van Roy and A. M. Despain. High-Performance logic programming with the Aquarius Prolog compiler. IEEE Computer, pages 54-67, Jan. 1992. 